\title{
Cervical cancer prevention in HIV-infected women in resource-limited settings
}

\section{Groesbeck Parham speaks to Head of Commissioning, Charlotte Barker}

Groesbeck Parham is Professor of Gynecologic Oncology and Infectious Diseases in the Department of Medicine at the University of Alabama at Birmingham (AL, USA) and Director of the Centre for Infectious Disease Research in Zambia's Cervical Cancer Prevention Program. A native Alabamian, Parham received his BA (1970) from Oberlin College, OH, USA, and medical degree (I98I) from the University of Alabama in Birmingham. He completed an obstetrics and gynecology residency (1985) at the University of Alabama in Birmingham, a urogynecology fellowship (1986) at the University of London, UK, and Khartoum Teaching Hospital, Sudan, and a gynecologic oncology fellowship (1988) at the University of California, Irvine, CA, USA. He is a board-certified gynecologic oncologist. Parham moved to Lusaka, Zambia, in 2005 to establish the Centre for Infectious Disease Research in Zambia's Cervical Cancer Prevention Program, which targets HIV-infected women. Before moving to Lusaka he served as director of the divisions of gynecologic oncology at Charles Drew University of Medicine and Science in Los Angeles, CA, USA, and the University of Arkansas for Medical Sciences in Little Rock, AR, USA. His primary research interest is the science of implementing cancer prevention technology in post-colonial Africa.

- How did you become involved in cervical cancer prevention in resource-limited settings? Deep down inside I am first and foremost a social activist, and have been so since I participated in the anti-apartheid (i.e., civil rights) movement in Birmingham, Alabama, in the early 1960s when I was 15 years old. But even before that I grew up in a poor working class community in an industrial area (Fairfield) around Birmingham, and as a child I observed the dignity and respect with which my mother and father treated people who were less fortunate than us (my father was a pharmacist and the proprietor of a small business). Having migrated from rural Alabama to Birmingham seeking employment opportunities related to the newly emerging steel industry in the 1920s, they both had an understanding of poverty and a heart for the poor and donated a significant amount of the profits from their business to pay the tuition of children from families that otherwise would not have been able to go to college. On many occasions in the 1950s, when I was only 7 or 8 years of age, I witnessed my mother opening our home to homeless women with no place to sleep on cold, rainy winter nights. It was very common for her to take food out of our refrigerator and share it with women in our neighborhood who were struggling to feed their children. So, my commitment to sharing with others and serving the poor was imprinted very early in my life.

My initial mentor, Dr Hugh Shingleton, former Head of the Division of Gynecologic Oncology at the University of Alabama when I was an obstetric/gynecology resident, opened the door to my initial international experience in medicine by giving me a chance to study abroad. In 1985, while studying urogynecology at the University of London, I met a Sudanese student who told me about a gynecologic surgeon in his country, Dr Abbo Hasaan Abbo, who repaired pelvic defects suffered by women who had undergone female genital mutilation as young girls. I cut my studies short in London and spent 3 months in Khartoum, Sudan, studying with Dr Abbo at the world-renowned Khartoum Fistula Hospital. That experience fundamentally changed my life because it showed me how one human being, committed to a single cause, even with very few resources, if willing to make personal sacrifices, can have a profound impact. From that point on I knew I had been 'called' to practice medicine in Africa, the only question was when.

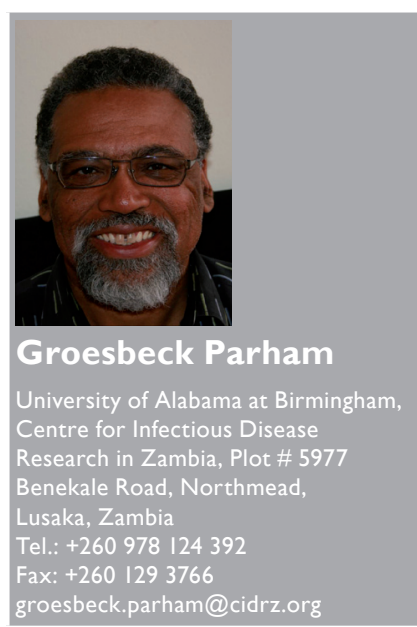


What are the main areas your research is focused on at present?

At present my primary area of research interest is the science and art of implementing, in an emerging African nation, a nurse-led, community volunteer-supported, point-of-care cervical cancer prevention program that uses: first, modern mobile technology for screening; second, online entry of data; and, third, cell phone call-based follow up (' $\mathrm{eC}$ ' electronic cervical cancer control).

Because of its long history of colonialism and neocolonialism, Africa lost control of its natural resources and the struggle to regain that control is somewhere in the distant future. Primarily, Africa remains a producer of raw materials, most of which it does not own, that are exported to more industrialized nations where they are processed into finished goods. Such a situation does not represent fertile ground for the development of science and technology. This is reflected in the very small percentage of the national budgets of African nations that are allocated for the development of science and technology. This, coupled with the flow of capital outside of countries to purchase finished products, has contributed to the intellectual brain drain. In medicine this is manifested in severe shortages of scientists and doctors, particularly those who are very highly skilled, such as immunologists, virologists, pathologists, medical oncologists, oncologic surgeons and radiation oncologists. Thus, the solution to the problem of cancer must be focused on prevention, and even there it will have to involve taskshifting of activities to nonphysicians and the appropriate use of modern, affordable mobile technology, such as the cell phone. At some point, to create the type of service model that will allow expansion of services in these environments, artificial intelligence will have to be incorporated to make up for the vast shortage of highly skilled personnel that it will take decades to train. An example would be computerized interpretation of mammograms, which is already state of the art in the USA. The key to all of this is how to use modern technology in a way that is appropriate for the environments and the cultures in which the new screening paradigms will be implemented.

A central component of arriving at these workable and sustainable solutions is getting our African colleagues involved from the point of conception and design, because it is they who really understand the need, the culture and how algorithms must be executed so as not to overburden healthcare infrastructures that are already overburdened. They must take the lead so as to create African solutions to African problems, but using the products of modern science and technology.

In the current issue of HIV Therapy, you publish effectiveness data from a large cervical cancer screening program in Zambia [1]: can you tell us a bit more about the aims \& significance of this program?

Over the past decade, a variety of evidencegenerating efforts (including randomized clinical trials) have demonstrated the utility of noncytological prevention methods such as visual inspection with acetic acid (VIA) and HPVDNA testing. These studies have shown the safety, acceptability, feasibility and effectiveness of the use of such methods in algorithms linking screening results to immediate cryotherapy ('screen and treat'), aimed at reducing cervical cancer incidence and mortality in the developing world. Yet, rarely have governments in low-income countries included any form of cervical cancer prevention in public sector systems that provide much of the healthcare for their populations.

We evaluated the effectiveness of 'screen and treat' prevention services for reducing cervical cancer incidence and mortality in a routine program implementation setting of the developing world, in HIV-infected women. That is what is novel and significant about our analysis.

Why are HIV-infected women more at risk of cervical cancer?

In general many of the risk factors for HPV and heterosexually acquired HIV are similar: unprotected early sexual intercourse, multiple sexual partners and sex with a partner who has multiple partners. However, HIV-infected women are at higher risk for developing cervical intraepithelial neoplasia and invasive cervical cancer, primarily because they have higher rates of persistent HPV infection due to HIVinduced impaired immunity. Additionally, the large majority of HIV-infected women in the world live in regions where there is little or no access to cervical cancer prevention services. This situation highlights the urgency of implementing national HPV vaccination and cervical cancer screening programs in global regions that are hardest hit by the HIV pandemic, the epicenter of which is sub-Saharan Africa. 
What were the main findings of this study? We were able to take a screening test that has moderate effectiveness but because it was implementable (i.e., adoptable), ended up having a high intervention to impact ratio: for every 46 HIV-infected women screened, one death from cervical cancer was prevented.

What lessons can we take from the program for large-scale HIV/public health interventions in resource-limited settings? Although screening modalities may have proven effectiveness in research studies and demonstration projects, unless widely implemented, they will ultimately have little impact on the target disease, or reduce morbidity and mortality in the at-risk populations (evidencebased effectiveness + lack of adoption $=$ no impact). Some key lessons in making them adoptable are:

- Choose a low-tech yet scalable prevention intervention modality;

- Horizontally integrate it with donor-funded HIV/AIDS care and treatment programs or other similar programs;

- Integrate into government-operated public health clinics;

- Apply setting-appropriate technology for quality control and telemedicine consultation;

- Link screening to treatment;

- Use local talent;

- Get on the ground;

- Partnerships should be African-led.

Where do you plan to focus your research over the next 5- 10 years?

There are large numbers of women who are already infected with HPV, who are well on the way to developing cervical precancers and cancers, so screening is mandatory. However, we can screen for the next 100 years and not stop this epidemic. We must move upstream and prevent women from becoming HPV-infected. That is why the HPV vaccination is very important, and getting a vaccination program set up is an immediate goal. The Zambian government has embraced the vaccine and we are now working with pharmaceutical companies to bring $\mathrm{HPV}$ vaccination into this country. Within the next $12-18$ months we hope to be rolling out a vaccination program for young girls.
Once we have the HPV vaccination program underway, and expand our cervical cancer prevention program nationwide, I plan to move on to a new area of research.

Using the perspective that is offered by the newly emerging field of psychoneuroimmunology I would like to try and unravel the mystery of the relationship between the human mind and cervical cancer (i.e., the effect of biobehavioral factors on cervical tumor biology). Epidemiologic studies indicate that stress, chronic depression and low self-esteem might serve as risk factors for cancer development and progression. The real question is what are the biologic processes that mediate such effects, and can we in some way intervene and thereby affect outcomes? Certain thoughts and life events, whether they are negative or positive, can apparently activate a cascade of information-processing pathways in the CNS, which stimulates fight or submission responses in the autonomic nervous system. Severe trauma and post-traumatic stress disorder are felt to be examples of stressors associated with alterations in the autonomic nervous system. It has been well-established that persistent infection with oncogenic HPV genotypes is a necessary cause of cervical cancer, but HPV infection alone is probably not sufficient to cause cancer. There are other co-factors that are a part of the cervical cancer story and have not yet been identified. My belief is that, in addition to lack of awareness and lack of screening and treatment opportunities, one of the reasons we see such high incidence of cervical cancer in sub-Saharan Africa and such advanced stages of the disease is because of the psychic trauma that African women have experienced and continue to experience, primarily due to the level of poverty in which they are forced to live. Some small clinical studies have already shown that negative responses to stressful life events are risk factors for both the development and progression of high-risk HPV infection and cervical dysplasia in HIV-infected women. It would be interesting to clinically investigate the relationship between chronic negative life events and their impact on the ability of HPV to evade cellular immune responses, and even more exciting to intervene with some form of culturally appropriate stress reduction to see if it alters the carcinogenic process. Such clinical investigations are now feasible given the availability of HPV testing and screening algorithms that permit the identification of cohorts of 
women who have HPV infection alone, with no evidence of clinical disease. To unravel these relationships in the face of HIV infection is even more challenging and intriguing.

\section{Financial \& competing interests disclosure}

The author has no relevant affiliations or financial involvement with any organization or entity with a financial interest in or financial conflict with the subject matter or materials discussed in the manuscript. This includes employment, consultancies, honoraria, stock ownership or options, expert testimony, grants or patents received or pending, or royalties.

No writing assistance was utilized in the production of this manuscript.

\section{Bibliography}

1 Parham GP, Mwanahamuntu MH,

Sahasrabuddhe VV et al.: Implementation of cervical cancer prevention services for HIV-infected women in Zambia: measuring program effectiveness. HIV Ther. 4(6), 713-722 (2010). 